\title{
Evaluation of inflammatory cytokines and oxidative stress markers in prostate cancer patients undergoing curative radiotherapy
}

\author{
PHEBE L. ABDEL-MESSEIH ${ }^{1}$, NEVEEN M. NOSSEIR ${ }^{I}$, OSAMA H. BAKHE \\ ${ }^{1}$ Atomic Energy Authority, Cairo, Egypt \\ ${ }^{2}$ Military Medical Academy, Cairo, Egypt
}

\begin{abstract}
Prostate cancer is the second leading cause of cancer-related death in men. The present study was carried out to investigate the radiation response of serum cytokines and oxidative markers to find out if these novel biomarkers have significant applications regarding radiation outcome in prostate cancer patients. Significant elevations of prostatic specific antigen (PSA), asymmetric dimethyl arginine (ADMA) and nitric oxide $(N O)$ were recorded in cancer prostate patients at the time of diagnosis compared to controls. Patients were subjected to radiotherapy post prostatectomy with a total dose of 66 Gy in 33 fractions (5 sessions/week) for 7 weeks. At the end of the seventh week post radiotherapy, ADMA levels were accentuated while the levels of PSA and NO were lower than before therapy. The level of inflammatory cytokines (interleukins IL-4, IL-5 and interferon-gamma) in post radiation therapy patients were significantly elevated compared to both controls and prostate cancer patients. A significant inverse correlation was observed in prostate cancer patients between ADMA and NO. Moreover, a significant inverse correlation in post radiation therapy patients was observed between IL-5 and PSA. These results are highly suggestive that there is a specific cytokine response in patients undergoing curative radiotherapy for prostate cancer.
\end{abstract}

Key words: prostate cancer, oxidative markers, serum cytokines.

(Cent Eur J Immunol 2017; 42 (1): 68-72)

\section{Introduction}

Prostate cancer (Pca) is the most commonly diagnosed malignancy in men [1]. Cancer prostate pathogenesis reflects both infectious agents, genetic variation, dietary carcinogens and hormonal imbalance leading to injury of prostate and to the development of chronic inflammation and regenerative risk factor lesions referred to as proliferative inflammatory atrophy (PIA) [2].

In the earliest stage, prostate cancer is curable with radical surgery or radiotherapy (RT) [3]. In the advanced stages adjuvant RT can be used following radical prostatectomy. Unfortunately, at present the ability to predict an individual Pca patient response to RT is limited and based mostly on pretreatment assessment of PSA, tumor stage (TNM) and pathologic Gleason score [4]. Radiation therapy mediates tumor cell killing primarily via apoptosis and often leads to necrosis and mitotic catastrophe due to the DNA damage evoked within the tumor microenvironment. Local RT on cancer cells occasionally induces the regression of metastatic cancer at distant sites, which have not been irradiated apparently through induction of adaptive immune responses.
This phenomenon has been called an abscopal effect and can be attributed to the induction and enhancement of endogenous, tumor innate and adaptive immune responses. Cytokines play an important role in the abscopal effect [5].

Prostatic specific antigen (PSA) is the most famous cancer biomarker among the general public and is accepted as the gold standard for Pca screening, prognosis and therapy monitoring. The use of PSA to monitor RT response is problematic because it can take a long time to reach the PSA nadir (i.e. the lowest value of PSA achieved post therapy) following RT. Moreover, the PSA profile can be complicated by multiple (often benign) bounces that eventually resolve and can be mistaken for cancer recurrence. Lastly, PSA-based monitoring provides no useful information regarding early or late radiation toxicities in normal tissues. There is increasing evidence that cancer monitoring with a single biomarker cannot reflect multiple information of cancer development and that using PSA alone is insufficient [6].

Asymmetric dimethyl-arginine (ADMA) is a small molecular weight substance of 202 Dalton and is an endothelial nitric oxide synthetase (e NOS) enz-catalyzing NO produc-

Correspondence: Phebe L. Abdel-Messeih, Atomic Energy Authority, Ahmed Elzomor - Nasr City, Cairo, Egypt, e-mail: febylotfy@yahoo.com Submitted: 15.01.2017; Accepted: 20.04.2016 
tion from L-arginine [7]. Nitric oxide (NO) is a key second messenger in most tissues where it is generated in a low concentration predominantly by the catalytic action of two constitutively expressed isoforms of nitric oxide synthetase (NOS). Both of these are found in tumors but malignancy is also associated with the expression of high levels of the inducible isoform of NOS which is responsible for high levels of NO concentration not associated with normal physiology [8]. NO is a multifaceted compound that may inhibit or promote carcinogenesis [9]. NO produces multiple effects that can influence the outcome of advanced cancer disease and metastasis. Specifically, NO regulates vasodilatation and platelet aggregation, which affect tumor angiogenesis. Moreover, the production of endogenous NO is associated with apoptosis of tumorigenic cells [10].

Cytokines are low-molecular weight proteins that regulate the immune system and inflammation by a complex but highly coordinated induction cascade [11]. Cytokines are major homeostatic modulators and may be associated with the pathophysiology of various types of cancers. Each cytokine forms a cytokine network and these networks may synchronize various biological events including immunity, cell differentiation, proliferation and cancer growth in humans [12].

Interleukin-4 (IL-4) is an important cytokine that plays a central role in regulating inflammatory and cell-mediated immune responses. It has a variety of functions including effects on hematopoietic tissues, tissue adhesion and inflammation. IL-4 exerts its function through activation of IL-4 receptor (IL-4 R $\alpha$ ) by tyrosine phosphorylation of Janus Family (Jak) tyrosine kinases, insulin receptor substrate proteins and signal transducers and activators of transcription [13]. Interleukin-4 and interleukin-5 act as immune-suppressors of cancer immunity and promote cancer development [14].

Interferon- $\gamma($ IFN- $\gamma)$ is a cytokine whose biological activity is conventionally associated with cytostatic/cytotoxic and antitumor mechanisms during cell mediated adaptive immune response. It has been used clinically to treat a variety of malignancies, despite evidence implicating a role of IFN- $\gamma$ in tumor immune surveillance; a steady flow of reports has suggested that it may also have pro-tumorigenic effects under certain circumstances [15].

The aim of this study is to evaluate the radiation response of serum cytokines and oxidative markers. These novel biomarkers have significant applications regarding radiation outcome in prostate cancer patients. The study represents the first attempt to study plasma cytokines in a well-defined group of patients receiving uniform pelvic radiotherapy.

\section{Material and methods}

The present study was carried on twenty male patients. Inclusion criteria included the candidacy for curative ra- diotherapy post prostatectomy from those attending the Radiation Oncology Center of Atomic Energy Authority. All tumors were completely inside prostate gland (PT 2 or PT 3) disease, no cancer cells in lymph nodes close to prostate (No) and no distant metastasis (Mo) according to the American Cancer Society Staging, no history of previous prostate surgery and consent to participate in the study. Exclusion criteria included candidates for any other treatments especially regarding nitric oxide producing drugs or suffering from any other disease especially inflammatory disorders. They were planned and received a total dose of $66 \mathrm{~Gy}$ in 33 fractions, 5 sessions/week for a period of 7 weeks. Quality assurance for imaging guided radiotherapy (R.T) was achieved by bony anatomy matching (Mean age was 72 years \pm 1.5 ). Samples were taken at the time of diagnosis before prostatectomy and after completion of Radiotherapy Sessions at the end of the seventh week. The control group consisted of twenty male subjects, their mean age was $72 \pm 1.2$. They had their routine visit at the preventive health service on the same day as patients. Sera from patients and controls were collected for the further biochemical analysis.

The procedures followed were in accordance with the ethical standards of the responsible committee on human experimentation and according to the ethical standards given in the Declaration of Helsinki, as revised in 2008.

\section{Biochemical analysis}

The level of Prostatic Specific Antigen (PSA) was determined by human Abcam's Prostate Specific Antigen PSA ELISA kit or also commonly termed tissue Kallikrein-3 (KLK3) [16]. Interferon $\gamma$ (IFN- $\gamma$ ) was determined by ELISA quantitative sandwich immunoassay kit provided from the Eagle Biosciences, Korea [17]. Interleukin 4 and 5 (IL-4 and IL-5) were determined using a highly sensitive fluorescence microsphere system (BioPlex suspension array system, Hercules, CA, USA) [18]. Serum ADMA levels were determined by enzyme linked immunosorbent assay (ELISA) using kits provided by Immundiagnostik AG, Germany [19]. Serum nitric oxide was measured as stable end product, nitrite, according to the method of Miranda et al. based on the reduction of nitrate by vanadium trichloride combined with detection by acidic Griess reaction [20].

\section{Statistics}

All values were expressed as mean $\pm \mathrm{SD}$, statistical analysis was carried out by one way analysis of variance (ANOVA) followed by Tukey-Kramer multiple comparison test. A $p$-value of 0.05 or less was taken as a criterion for a statically significant difference, and correlation coefficient were used to evaluate the effects of the studied groups compared with the control group [21]. 


\section{Results}

The physical characteristics and laboratory investigations in the studied groups including prostate cancer patients at diagnosis and at the end of radiotherapy compared to controls are demonstrated in Table 1.

The golden standard tumor marker PSA and oxidative status markers (ADMA and NO) were significantly elevated in cancer prostate patients at the time of diagnosis compared to controls. Post radiation therapy patients had elevated ADMA levels compared to both controls and cancer prostate patients at the time of diagnosis. PSA and NO levels decreased compared to the levels before therapy, however, still significantly higher than controls. A comparison of levels of inflammatory cytokines in the studied groups are illustrated in Table 2.

Regarding inflammatory cytokines (IL-4, IL-5 and IFN- $\gamma$ ) in post radiation therapy patients, levels were significantly elevated compared to both cancer prostate patients controls. Cancer prostate patients at the time of diagnosis had significantly elevated IL-4 levels and a significantly lowered IFN- $\gamma$ level compared to controls. Figure 1 shows that post radiation therapy patients had a significant inverse correlation between IL-5 and PSA $\left(r^{2}=0.82\right)$. Figure 2 illustrates a significant negative correlation between ADMA and NO in cancer prostate patients $\left(r^{2}=0.81\right)$.

\section{Discussion}

Prostate cancer is the most common cancer in men. About 20 percent of all human cancers are caused by chronic infection or chronic inflammatory states. Radiation therapy is one of the main modalities used to treat localized prostate cancer at the present time. Radiation induced protein expression is dose dependent and can be cell-type specific in the case of individual proteins such as PSA [22]. New biomarkers are needed in radiation oncology to optimize Pca decision making, treatment and therapy

Table 1. Characteristics and laboratory investigations of the studied groups

\begin{tabular}{lccc}
\hline Parameter & $\begin{array}{c}\text { Control } \\
(\boldsymbol{n}=\mathbf{2 0})\end{array}$ & \multicolumn{2}{c}{ Prostate Cancer $(\boldsymbol{n}=\mathbf{2 0})$} \\
\cline { 3 - 4 } & & At Diagnosis & $\begin{array}{c}\text { Post } \\
\text { Radiotherapy }\end{array}$ \\
\hline Age (years) & $72 \pm 1.2^{\mathrm{a}}$ & $72 \pm 1.5^{\mathrm{a}}$ & $72 \pm 1.5^{\mathrm{a}}$ \\
\hline PSA $(\mathrm{ng} / \mathrm{ml})$ & $0.36 \pm 0.17^{* \mathrm{a}}$ & $202 \pm 4.68^{* \mathrm{~b}}$ & $137.9 \pm 5.36^{* \mathrm{c}}$ \\
\hline $\begin{array}{l}\text { ADMA } \\
(\mu \mathrm{mol} / \mathrm{l})\end{array}$ & $0.68 \pm 0.13^{\mathrm{a}}$ & $0.9 \pm 0.13^{\mathrm{b}}$ & $1.17 \pm 5.36^{\mathrm{c}}$ \\
\hline $\begin{array}{l}\mathrm{NO} \\
(\mathrm{nmol} / \mathrm{ml})\end{array}$ & $40.6 \pm 3.15^{\mathrm{a}}$ & $53.4 \pm 2.48^{\mathrm{b}}$ & $46.7 \pm 2.15^{\mathrm{c}}$ \\
\hline $\begin{array}{l}\text { Values with a differen } \\
\end{array}$ & & \\
\hline
\end{tabular}

$\overline{\text { Values with a different superscript in the same row are significantly different }}$ at $p<0.05$

$*$ Highly significant $(p<0.001)$ monitoring for individual patients. Numerous cytokines, growth factors, and every other proteins are systematically up-regulated in response to cancer progression and treatment [23].

There is increasing evidence that cancer monitoring, in patients on radiotherapy, with a single biomarker cannot reflect total tumor mass and that using PSA alone may be insufficient in this regard [24].

PSA is the golden standard biomarker for monitoring tumor radiation response. However, given that patients exhibit a heterogeneous array of responses to radiotherapy, novel markers would be useful to monitor tumor and normal tissue response to treatment [25]. Data of the present study revealed that PSA levels increased post radiation compared to the controls. The same results were observed by Duke et al., who reported that PSA increased post radiation followed by a plateau [26]. And also supports the finding of Lee et al. [9].

ADMA is the endogenous inhibitor of endothelial nitric oxide synthetase (eNoS); enzyme catalyzing NO production from L-arginine [7]. In the present study, ADMA levels were significantly elevated in prostate cancer patients at the time of diagnosis and at post radiation therapy. Nitric oxide (NO) was significantly elevated at the time of diagnosis of prostate cancer. Data suggest that NO production is a factor that contributes to the death of circulating tumor cells and that NO plasma levels may contribute to the control of disease progression [10]. This may be the cause of decreasing NO post-radiotherapy compared to that at diagnosis. NO promotes growth rate and invasiveness of tumors and have a role in monitoring tumor blood supply in prostate cancer patients. The present study showed a significant inverse correlation between ADMA and NO.

There is growing evidence that the immune system, and in particular cytokines, play an important role in prostate cancer immunosurveillance and progression [3]. A limited number of clinical studies have shown that radiation induces interleukins and other pro-inflammatory cytokines,

Table 2. Comparison of levels of inflammatory cytokines in the studied groups

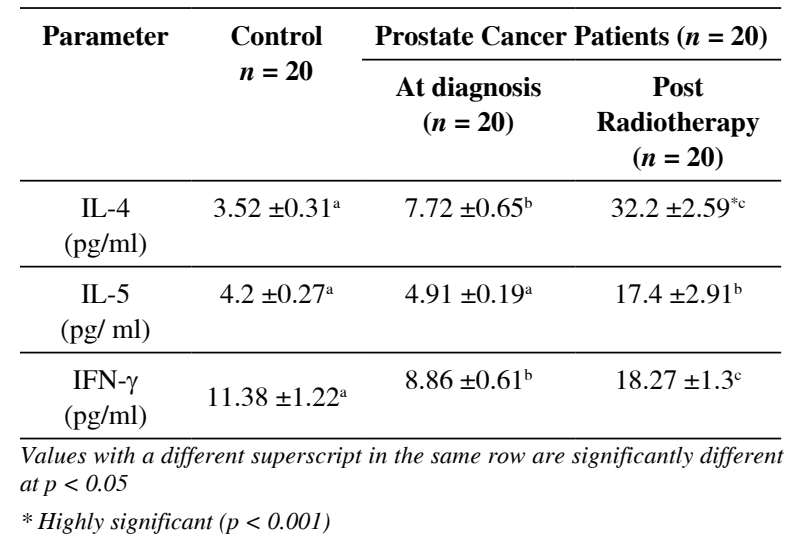




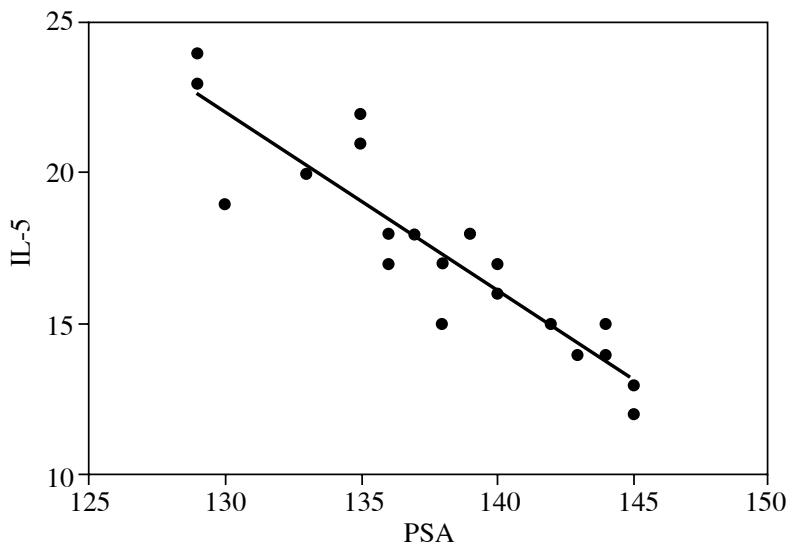

Fig. 1. Correlation between IL-5 and PSA after radiotherapy $\left(r^{2}=0.82\right)$

and that these proteins play a major role in mediating radiation responses [27].

Interleukin 4 and 5 (IL-4, IL-5) are important cytokines that play a central role in regulating inflammatory and cell-mediated immune responses. In accordance with the results of Ueda et al., it was demonstrated in this study that serum levels of IL-4 increased in Pca patients. Moreover, the present study showed significantly higher interleukin levels (IL-4 and IL-5) post radiation therapy compared to controls and prostate cancer patients. IL-4 and IL-5 are representative T-helper 2 cytokines that may be associated with cancer development. In particular, they act as immunosuppressors of cancer immunity and promote cancer development [14]. Similar results were obtained by Christensen et al. who concluded that cytokines are up-regulated in response to radiation and that there is no latency period for the acute and late molecular changes that occur subclinically involving changes in gene expression. Goldstein et al. showed that IL-4 is a pleiotropic cytokine produced largely by activated Th-2-polarized T-cells, mast cells and basophils. It acts upon a broad range of targets including hematopoietic cells, endothelial cells and tumor cells. One mechanism by which IL-4 appears to enhance the survival of tumor cells is through a direct anti-apoptotic effect. Emerging evidence indicates that IL-4 also exerts pro-tumorigenic indirect effects mediated via the tumor microenvironment [3].

Tazaki et al. reported that immunosuppressive cytokines (IL-4 and IL-5) were significantly elevated in Pca and may have contributed to further cancer development [14]. The present study documented a significant inverse correlation between PSA and IL-5 in the post radiation therapy group. Ueda et al. found that IL-4 levels are directly correlated with elevated levels of serum PSA [13]. However, Tazaki et al. did not find any correlation between serum PSA and serum cytokine levels [14].

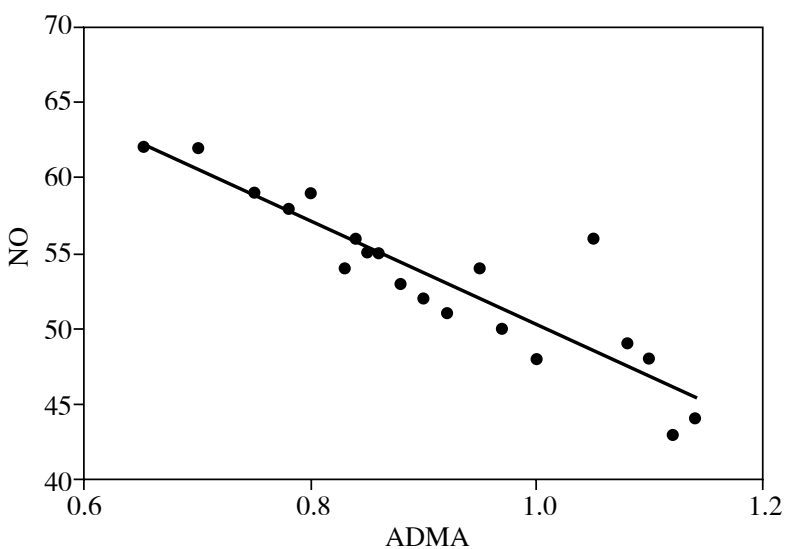

Fig. 2. Correlation between ADMA and NO in cancer prostate patients at diagnosis $\left(r^{2}=0.81\right)$

Recent studies have demonstrated the central involvement of helper T cells (Th) in anti-tumor immunity. In general, the cell responses tend to be polarized to either Th1-like or Th2-like phenotype based on the pattern of cytokines secreted. The (Th1) phenotype typified by secretion of lymphotoxin, tumor necrosis factor $\alpha$ (TNF- $\alpha$ ) and IFN- $\gamma$ have been more associated with cellular immunity and proved to be protective against development of certain types of intracellular pathogens and in eliciting antitumor responses [28]. Interferon $\gamma$ is a cytokine whose biological activity is conventionally associated with cytostatic/cytotoxic and antitumor mechanisms during cell mediated adaptive immune response. In accordance with results of the present study, Christensen et al. observed that IFN- $\gamma$ significantly increased during radiotherapy [25]. Our results reported significantly higher interferon-gamma levels post radiotherapy compared to both controls and prostate cancer patients. Also Tazaki et al. reported elevated INF- $\gamma$ in prostate cancer patients [14]. Dong et al. reported that the in vitro treatment of tumor cells with cytokines such as IFN- $\gamma$ induces production of NO, which leads to apoptosis and both sequelae could be blocked by specific inhibition of NO production [29]. Another explanation of the role of INF- $\gamma$ antitumor mechanism was proposed by Fang et al. who documented that interferon gamma can inhibit cell proliferation and apoptosis predominantly through Jak/STAT1 pathway [30].

\section{Conclusions}

The results obtained suggest that there is a specific cytokine response in patients undergoing curative radiotherapy for prostate cancer.

The authors declare no conflict of interest. 


\section{References}

1. Garcia-Hernandez M, Gray A, Hubly B, et al. (2008): Prostate Stem cell antigen vaccination induces a long term protective immune response against prostate cancer in the absence of autoimmunity. Cancer Res 68: 861-869.

2. Marzo A, Platz EA, Sutcliffe S, et al. (2007): Inflammation in Prostate Carcinogenesis. Nature Rev Cancer 7: 256-269.

3. Goldstein R, Hanley C, Morris J, et al. (2011): Clinical investigation of the role of interleukin- 4 and interleukin-13 in the evoluation of prostate cancer. Cancers (Basel) 3: 4281-4293.

4. Nicol AM, Warde P, Bristow RG (2005): Optimal treatment of intermediate-risk prostate carcinoma with radiotherapy. Cancer 104: 891-905.

5. Park B, Yee C, Lee KM (2014): The Effect of Radiation on the Immune Response to Cancers. Int J Mol Sci; 15: 927-943.

6. Merrick G, Butler W, Wallner K, et al. (2002): PSA Spikes after Permanent Prostate Branchy Therapy. Int J Radiat Oncol Biol Phys; 54: 450-456.

7. Yukawa S, Hibino A, Maeda T (2002): Nephrology Dialysis Transplantation. 17: 88-109.

8. Hirst D, Robson T (2010): Nitric oxide in cancer therapeutics: interaction with cystolic chemotherapy. Curr Pharm Des 16: 411-420.

9. Lee KM, Kang D, Park SK, et al. (2009): Nitric oxide synthase gene polymorphism and prostate cancer risk. Carcinogenesis 30: 621-625.

10. Medeiros RM, Morais A, Vasconcelos A, et al. (2002): Outcome in prostate cancer: association with endothelial nitic oxide synthase Glu-Asp 298 polymorphism at exon 7. Clin Cancer Res 8: 3422-3437.

11. Goldsby R, Kindt TJ, Osborne BA (2002): Cytokines. In: Kuby Immunology. 4th edition. W.H. Freeman and Company, New York; 303-327.

12. Kuroda K, Nakashima J, Kanaa K, et al. (2007): IL-6 is associated with cachexia in patients with prostate cancer. Urology 69: 113-117.

13. Ueda T, Sadar M, Suzuki H, et al. (2005): IL-4 in patients with prostate cancer. Anticancer Res 25: 4595-4598.

14. Tazaki E, Shimizu N, Kimura H (2011): Serum cytokine profile in patients with prostate cancer. Exp Ther Med 2: 887891.

15. Zaidi MR, Merlino G (2011): The two faces of interferon $\gamma$ in cancer. Clin. Cancer Res 17: 6118-6124.

16. Banville N (2014): A quantitative proteomic approach significantly altered protein networks in the serum of patients with lymphangioleiomatosis. PLoS One 9: e 105365.

17. Abdel-Latif M, Sakran T, El-Shahawi G, El-Fayoumi H (2015): Immunomodulatory effect of diethylcarbamazine citrate plus filarial excretory-secretory product on rat hepatocarcinogenesis. Int Immunopharmacol 24: 173-181.

18. Lequin RM (2005): Enzyme immunoassay enzyme linked immunosorbent assays. Clin Chem 51: 2415-2418.

19. Nijveldt RJ, Teerlink T, Vander Hoven B, et al. (2003): Asymmetrical dimethylarginine (ADMA) in critically ill patients: high plasma ADMA concentration is an independent risk factor of ICU mortality. Clin Nutr; 22: 23-30.

20. Miranda K M, Espey MG,Wink DA (2001): A rapid, simple spectrophotometric method for simultaneous detection of nitrate and nitrite. Nitric Oxide 5: 62-71.

21. Spearman C (1904). The proof and measurement of association between two things. Am J Psychol; 15: 72.
22. Borgono CA, Diamandis EP (2004): The Emerging Roles of human Tissue kallikrins in cancer. Nature Review Cancer 4: 876-890.

23. Ray ME, Thames HD, Levy LB, et al. (2004): PSA nadir predicts biochemical and distant failures after external beam radiotherapy for prostate cancer. International J Radiat Oncol Biol Phys 64: 1140-1150.

24. Veenstra T, Prieto D, Conrads T (2004): Proteomic patterns for early cancer detection. Drug Discovery Today 9: 889-897.

25. Christensen E, Evans KR, Menard C, et al. (2008): Practical approaches to proteomic biomarkers within prostate cancer radiotherapy trials. Cancer Metastasis Rev 27: 375-385.

26. Duke JP, Zakian KL, Spees WM, et al. (2003): Metabolic response of the CWR 22 prostate tumor xenograft after 20 $\mathrm{GY}$ of radiation studied by $1 \mathrm{H}$ spectroscopic imaging. Clin Cancer Res 9: 4529-4536.

27. Hall EJ (2000): Clinical Response of Normal Tissue. In: Radiobiology for the Radiologist. 5th edition. Lippincott Williams \& Wilkins, New York; 339-360.

28. Egeter O, Mocikat R, Ghoreschi K, et al. (2000): Eradication of disseminated lymphomas with Cp G-DNA activated T-helper type 1 cells from nontransgenic mice. Cancer Res 60: 1515-1520.

29. Dong Z, Staroselsky A, Qi H, et al. (1994): Inverse correlation between expression of inducible nitric oxide synthase activity and production of metastasis in K-1735 murine melanoma cells. Cancer Res 54: 789-793.

30. Fang P, Hwa V, Little BM, et al. (2008): IGFBP-3 Sensitizes Prostate Cancer Cells to Interferon Gamma Induced Apoptosis. Growth Horm IGF Res 18: 38-46. 